\title{
VLBI STRUCTURE OF NGC 1275 AT 3 MILLIMETERS
}

Melvyn Wright, Radio Astronomy Laboratory, UC Berkeley

We have made $\lambda 3 \mathrm{~mm}$ VLBI observations of $3 \mathrm{C} 84$ at 6 epochs between 1981 and 1987, using 6m antennas at the Hat Creek Radio Astronomy Observatory (HCRK), 10m antennas at the Owens Valley Radio Observatory (OVRO), the 12m Kitt Peak telescope (KTPK), and the $14 \mathrm{~m}$ telescope at the Five College Radio Astronomy Observatory (QBBN). Model fitting suggests a core-halo structure, which we associate with the active nucleus of NGC 1275. The unresolved core, which is smaller than $0.1 \mathrm{pc}$ $\left(0.2\right.$ mas; $\mathrm{z}=0.018 ; \mathrm{H}_{0}=50 \mathrm{~km} \mathrm{~s}^{-1} \mathrm{Mpc}^{-1}$ ) shows a slow decay corresponding to the decrease in total flux density since the flare in 1980. In 1985 we resolved the core component on a transcontinental baseline $\left(10^{9} \lambda\right)$ into a compact nucleus, < 0.1 mas, and a "jet" extended at a position angle $205^{\circ}$ and unresolved in width, < 0.1 mas. There is also evidence for a compact emission region located 10 mas from the nucleus at a position angle $170^{\circ}$. This structure corresponds closely with that observed at the same epoch in VLBI maps at $22 \mathrm{GHz}^{1,2}$.

The $\sim 100 \mu$ arcsec resolution provided by VLBI observations at $\lambda 3 \mathrm{~mm}$ on transcontinental baselines can probe the broad line emission and jet forming regions of quasars and the scale of an accretion disk around massive black holes in nearby active galaxies. Millimeter observations are required to probe the optically thick synchrotron components seen at centimeter wavelengths. For Sgr $A$ * the measured size scales as $\lambda^{2}$; observations at $\lambda$ 3mm provide a resolution $\sim 1 \mathrm{AU}$. The small size of millimeter telescopes $(6-14 \mathrm{~m})$, relatively high system temperatures $(300-500 \mathrm{~K})$ and tropospheric turbulence limit on integration times $(\sim 100 \mathrm{~s})$ result in poor sensitivities. The limited uv coverage currently restricts our analysis to model fitting. Table 1 summarizes the experiments to date.

The peak flux density of the $3 \mathrm{C} 84$ flare was $45 \mathrm{Jy}$ in early $1980^{3}$. The brightness temperature must be less than $\sim 10^{12} \mathrm{~K}$, corresponding to the inverse Compton limit; thus the minimum size in $1980 \sim 0.1$ mas. The magnetic field, estimated from optically thick sychrotron emission, $\sim 1$ Gauss. The equipartition size is also $\sim 0.1$ mas. The core in our model-fit is smaller than 0.2 mas. If this core were the site of the $\mathrm{mm}$ flare then the emitting region did not grow in size by more than $0.15 \mathrm{ly}$ in five years, as the flux density decayed. We suggest that the 1980 flare came from a wind or jet whose size at unity optical depth is proportional to the observed intensity which, in turn, is a measure of the relativistic particle injection rate. Future millimeter VLBI observations during a flare are needed to confirm this hypothesis. The observed decay of emission is not consistent with a single outburst in a magnetic field $>\sim 0.1$ Gauss. 
There was a 208 increase in total flux density in 1984; this increase was not reflected in any of the compact components detected in the VLBI experiments. Our measurements of 3C345, detected in 1982, 1984 and 1985 supports our flux density calibration ${ }^{4}$. The data for 3C84 imply that the increase in total flux density in 1984 was due to a component larger than $\sim 0.5 \mathrm{pc}$. This flare may result from secondary particle generation in a shock. The primary event may well be the flare in 1980; no other precursors are obvious in the data. Note that, if the details in the total flux density variations correspond to secondary particle generation, then we would not necessarily expect these details to be reflected in the evolution of the compact VLBI components.

We can look forward to improved sensitivity and 'x coverage in the near future. Experiments phasing the antennas at HCRK and OVRO have been successful and use of a lens at HCRK has increased the aperture efficiency to 808 . A greatly improved uv coverage can be obtained by observing at 3 frequencies, 80 to $110 \mathrm{GHz}$, with existing antennas, and we can look forward to more antennas and increased bandwidth with the VLBA. Millimeter VLBI has a bright future.

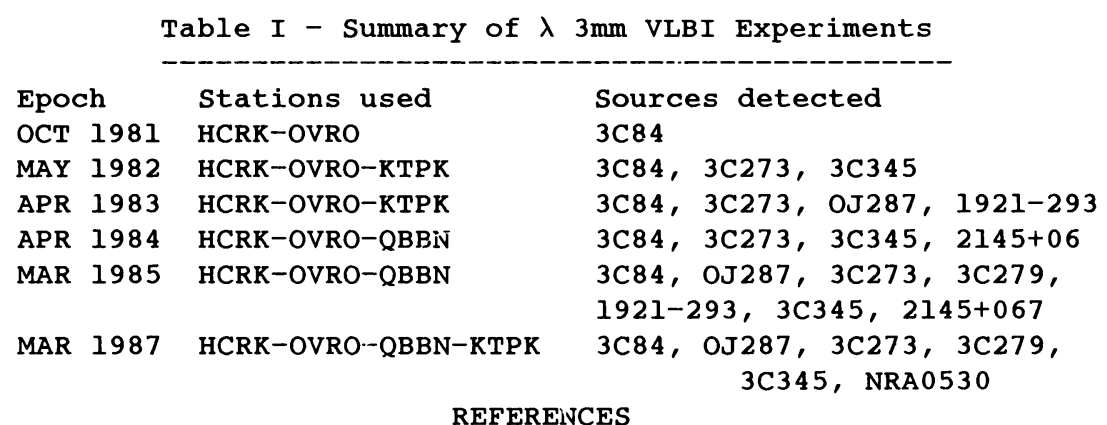

1. A.C.S.Readhead, D.H.Hough, M.S.Ewing, R.C.Walker and J.D.Romney, 1983, Ap.J., 265, 107.

2. J.M.Marr, D.C.Backer, M.C.H.Wright, and A.C.S.Readhead, These proceedings.

3. W.A.Dent, C.P.O'Dea, T.J.Balonek, R.W.Hobbs, and R.J.Howard, 1983, Nature, 306, 41 .

4. D.C.Backer, M.C.H.Wright, R.L.Plambeck, J.E.Carlstrom, C.R.Masson, A.T.Moffet, A.C.S.Readhead, D.Woody, A.E.E.Rogers, J.M.Moran, C.R.Predmore, and R.L.Dickman, 1987, Ap.J., 322, 74. 